\title{
An in vitro genotoxicity study of silver amalgam on Ames test
}

\author{
A. Hassan \\ S.A. Omar \\ Z. Ariffin \\ School of Dental Sciences, Health Campus, Universiti Sains Malaysia \\ Kelantan, Malaysia \\ E-mail: drakramhassan@yahoo.com \\ Received August 15, 2010; Accepted September 20, 2010
}

\begin{abstract}
Silver amalgam/Silverfil Argentum ${ }^{\circledR}$ is a 'Malaysian made amalgam' has already been approved to be free from cytotoxicity, however its genotoxic effect has not been explored yet as biocompatible material. The objective of this study was to identify the genotoxic characteristic of silver amalgam by using Bacterial Reverse Mutation Assay (Ames test). This was a descriptive experimental study involving one strain of mutated Salmonella. The test material was evaluated in one mutated strain of Salmonella typhimurium TA1538 with and without an external metabolic activation system (S9 Mix). The bacteria were incubated for 48 hours at $37 \pm 0.5$ 으 before the colony growth or revertant colonies were counted. Data obtained were analyzed by using non-statistical method. The investigation of the genotoxic reaction on the test material revealed that the number of revertant colonies in both strains with and without S9 Mix were less than twice of the negative control even in the presence of high silver amalgam concentrations $(5.0 \mu \mathrm{g} / \mathrm{ml})$. This study demonstrated that the test material did not exhibit any mutagenic activity under the chosen conditions. Thus, silver amalgam could be considered to have no genotoxicity effect.
\end{abstract}

Keywords: Silver amalgam, genotoxicity, Ames test

\section{Introduction}

Dental amalgam was recognized to be the most long lasting restorative material in dentistry ever been made until now. In spite of good hardness properties, level of toxicity of mercury content is keep declining over the years. Many companies of amalgam product passionately running in research and put full effort in order to notify their product to be free from mutagenesis.

Silver amalgam/Silverfil Argentum is a Malaysian made amalgam developed since 13 years ago has successfully received FDA 510 (K) clearance for cytotoxicity from the United States Department of Health and Services in America ${ }^{1}$.

An international standard ${ }^{2}$ lays down specific requirements for biocompatibility, including the tests based on the nature of the contact and the duration of implantation of the biomaterial. The standard stipulates that all materials that will be in contact with mucous membrane, bone or dentinal tissue where the contact exceeds 30 days, as well as all implantable devices where the contact exceeds 24 hours, must undergo genotoxicity testing ${ }^{3}$. 
According to ISO 10993-3 [2] when the genetic toxicity of a medical device has to be experimentally assessed, a series of in vitro tests shall be used, this series shall include at least three assays and at least two of these should preferably use mammalian cells as a target.

Kirkpatrick et al. ${ }^{4}$ and Katzer et al. ${ }^{5}$ suggested using an in vitro study after allowing for cost intensive and time consuming. Moltelmans and Zeiger ${ }^{6}$ strongly agreed that Salmonella typhymurium/ microsome assay to be widely accepted as a shortterm bacterial assay in order to identify the substance that can produce genetic damage that leads to gene mutations.

To reduce the number of biomechanical studies, evaluation of new biomaterials should nowadays begin with in vitro cytotoxicity and mutagenicity tests. This applies for the development of both temporary and permanent implants and prostheses and for permanent implant particularly under the aspect that carcinogenic potential is often the consequence of chronic exposure to minute concentrations ${ }^{5}$.

Although silver amalgam had successfully explored in cytotoxic effect studies, the genotoxic characteristics have to be carried out before it can be known as biocompatible material.

Therefore, the aim of this study was to identify the genotoxicity effect of silver amalgam by using Bacterial Reverse Mutation Assay (Ames test).

\section{Material and Methods}

\subsection{Study design and sample size}

This was a descriptive experimental study using Ames test. The test was conducted in Ames laboratory in School of Dental Sciences, Universiti Sains Malaysia. No sample size required in this study. Replication of 2-3 plates for each positive \& negative control and the test substance with different doses with and without metabolic activation (S9 Mix) were taken base on previous study ${ }^{7}$. The Salmonella typhimurium which was then stored as frozen stock culture.

\subsection{Test substance}

Silver amalgam/ Silverfil Argentum was supplied by Dunia Perwira Manufacturing Sdn. Bhd. and available in capsule form. In preparation of the tested material, the capsule containing silver amalgam was triturated by using amalgamator for 5 seconds. The bolus was let to be dried and hardened before it could be powderized. The powder was weighted into 5 unit of scale of $5000 \mu \mathrm{g} / 2500 \mu \mathrm{g} / 1250 \mu \mathrm{g} / 625 \mu \mathrm{g} / 313 \mu \mathrm{g}$ per plate respectively by using micro weight machine (mg scale). Each unit scale of powder was then diluted into $1 \mathrm{ml}$ of distilled water and kept inside incubator for 24 hours at $37 \pm 0.5^{\circ} \mathrm{C}$ rolling prior to the procedure.

\subsection{Negative and positive controls}

The negative control used in this study was sterile distilled water ${ }^{8}$. Specific positive controls were used in order to confirm the reversion properties and the specificity of each tester strain and the efficacy of the metabolic activation system. Toxic positive control application caused genotoxic effect (reverse mutation) to bacterial strains. Positive control chemicals were specifically designed as highly mutagenic to each strain. For strain TA1538, 4-Nitroo-phenylenediamine was applied without S-9 Mix (which was replaced by sodium phosphate buffer). On other hand, 2- Aminoantracene (2AA) was used in S-9 Mix inclusion ${ }^{6}$.

\subsection{Tester strain}

Basically, this test uses a number of Salmonella strains with preexisting mutation that leave the bacteria unable to synthesize the required amino acid, histidine and therefore unable to grow onto histidine-free agar. Once being exposed to the mutagens, it will revert and allow the cells to synthesized histidine and become histidine independent that make it able to grow and form a colony on the same plate ${ }^{5}$. Here, TA1538 was selected strains used under samples 471 approved by International Organization for Standardization ${ }^{2}$. 
Genetically, TA1538 was going to be for detection of frameshift mutation [8]. This strain was obtained from Dr. T. Nohmi from National Institute of Health Science, Tokyo, Japan and was stored as frozen stock cultures $(0.035 \mathrm{ml}$ of DMSO / $0.4 \mathrm{ml}$ of broth culture) at $-80 \pm 5^{\circ} \mathrm{C}$ in ultra-deep freezer (MDF 392AT, SANYO). Therefore, reviving this two strain before use are very important particularly for genetic analysis.

Reviving or frozen working culture process began with inoculation of $1 \mathrm{ml}$ of Salmonella typhimurium into $5 \mathrm{ml}$ of nutrient broth and a nutrient agar plate. This procedure was done in a 'Lamina Flow'. This two medium was then incubated for overnight in the incubator (SANYO) at $37 \pm 0.5^{\circ} \mathrm{C}$. The result was shown cloudy-look in nutrient broth and colonies were formed on the nutrient agar. One isolated healthy colony was transferred and streaked onto Glucose Minimal (GM) agar and incubated for 48 hours at $37 \pm 0.5^{\circ} \mathrm{C}$. This would later produced colony and subculture was repeated for another 48 hours. The last colony formed on the GM agar so-called master plate was being run for genetic analysis by streaking the bacteria onto histidine, biotin and histidine-biotin filled agar for 15 hours $^{6-8}$. There was no growth seen in histidine and biotin agar but presence on histinebiotin agar. rfa marker had shown growth inhibition which proved that TA1538 was mutated on specific genotype. After underwent genetic analysis, TA1538 was ready to be used.

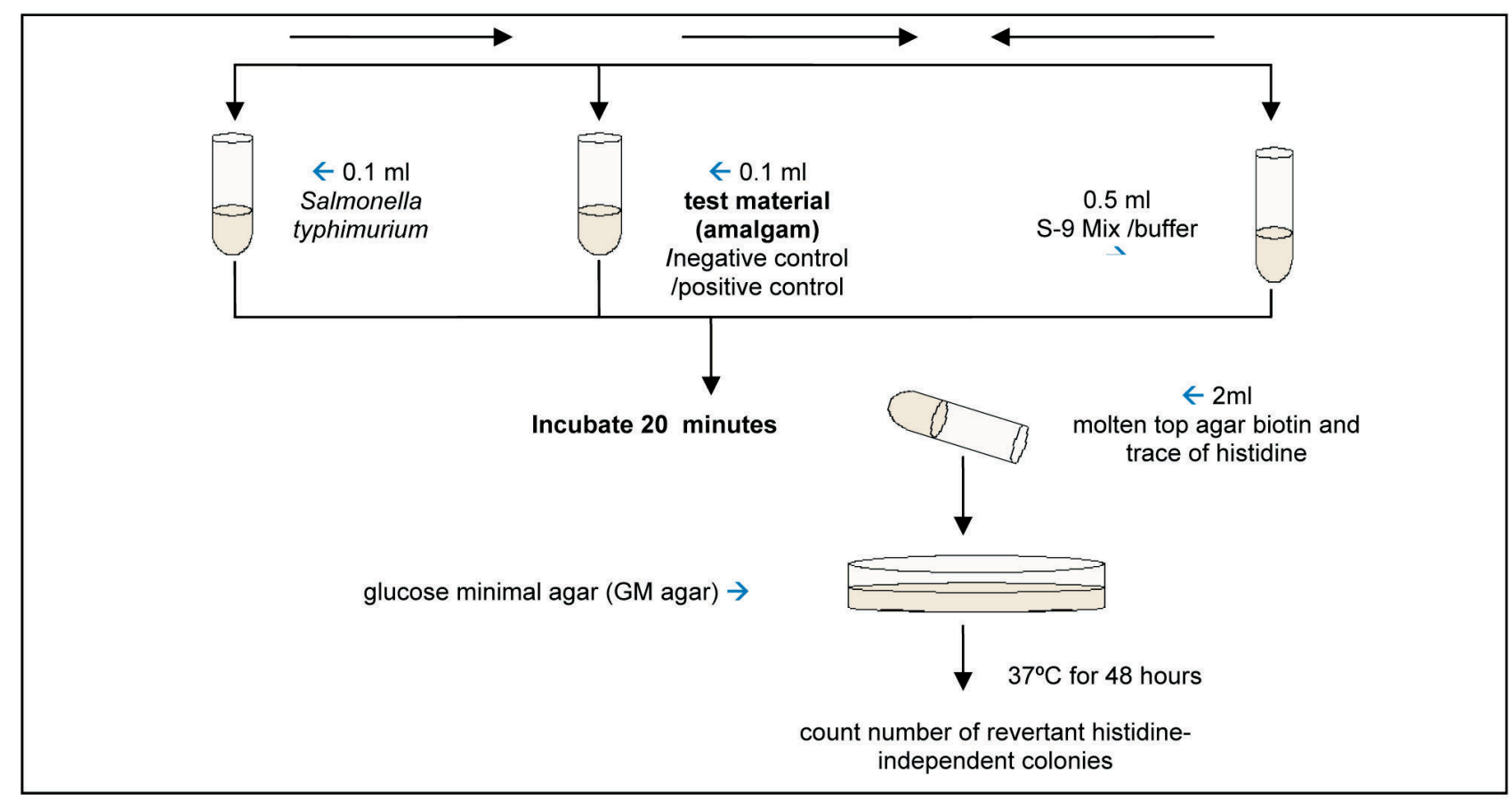

Figure 1. Procedure for Ames test 


\subsection{Medium and S9 Mix}

Minimal glucose agar plate was supplied by Oriental Yeast Co. Ltd., Japan and consisted of following components: X20 Vogel-Bonner minimum medium E $(50 \mathrm{ml} / \mathrm{L}), 40 \mathrm{w} / \mathrm{v} \%$ glucose $(50 \mathrm{ml} / \mathrm{L})$ and Agar (Bacto-Agar, Difco Laboratories) (15g/L). Soft agar containing $0.5 \mathrm{mM}$ histidine and $0.5 \mathrm{mM}$ biotin for S. typhimurium strains or $0.5 \mathrm{mM}$ tryptophan for $E$. coli strain was added to soft agar solution containing $0.6 \mathrm{w} / \mathrm{v} \%$ agar ( Bacto-Agar, Difco Laboratories ) and $0.5 \mathrm{w} / \mathrm{v} \% \mathrm{NaCl}$ in a ratio of 1:10. S9 Mix was supplied by Kikkoman Co. Ltd., Japan. One $\mathrm{ml}$ of S9 Mix contains protein $(25.19 \mathrm{mg} / \mathrm{ml}$ ), cytochrome P-450 $(0.97 \mathrm{nmol} / \mathrm{mg}$ protein), DMN demethylase activity ( $4.97 \mathrm{nmol} \mathrm{HCHO}$ formed/mg protein/min), aniline hydroxylase activity ( $24.02 \mathrm{nmol} \mathrm{p}$-aminophenol formed/mg protein/hr) and B [a] P hydroxylase activity ( 16.85 times higher than non induced S9).

\subsection{Procedures}

After $0.1 \mathrm{ml}$ of the test substance solution, $0.5 \mathrm{ml}$ of $0.1 \mathrm{M}$ sodium phosphate buffer ( $\mathrm{pH} 7.4$ ) or S9 Mix and $0.1 \mathrm{ml}$ of the bacterial culture were added to a tube, the mixture were incubated 20 minutes at 37 $\pm 0.5^{\circ} \mathrm{C} .2 \mathrm{ml}$ of soft agar was then added to each tube and poured onto a minimal glucose agar plate. After incubation for 48 hours at $37 \pm 5^{\circ} \mathrm{C}$, the number of revertant colonies was counted. As for the sterility test, $0.1 \mathrm{ml}$ of test substance solution, $\mathrm{S} 9 \mathrm{Mix}$ and $0.1 \mathrm{M}$ sodium phosphate buffer $(\mathrm{pH}$ 7.4) were poured onto a minimal glucose agar plate and incubated at $37 \pm 0.5^{\circ} \mathrm{C}$ for 48 hours to check for the bacterial contamination. Pure water was used as negative control and the following positive controls were used for bacterial strain.

\subsection{Colony counting}

The number of colonies were counted with a manual counter or a colony analyzer ( ProtoCol). Each plate was counted three times and the average of the three counts was adopted as the number of revertant colonies on the plate. The average number of revertant colonies for each dose was calculated as the average plate count for a set of duplicate. Decimals of the average figures were rounded off.

\subsection{Interpretation of the results}

Signs of toxicity or growth inhibition for all the bacterial strains under the test condition was described in this report. The test substance was judged to be negative, when the number of revertant colonies was less than twice that of the negative control.

\subsection{Analysis (Non Statistical Procedures)}

For this study, a non-statistical procedure was used to evaluate the results of Salmonella experiments [6]. Positive: A compound is considered a mutagen if it produces a reproducible, dose-related increase in the number of revertant colonies in one or more strains. A minimum fold increase, usually $2-3$ fold, in revertants (over the solvent control) is the cutoff between a mutagenic and nonmutagenic response. A compound is considered a weak mutagen if it produces a reproducible, dose-related increase in the number of revertant colonies in one or more strains but the number of revertants is not double of the background. Negative: A compound is considered a nonmutagen if no dose-related increase in the number of revertant colonies is observed in at least two independent experiments. Inconclusive: If a compound cannot be identified clearly as a mutagen or a nonmutagen, the results are classified as inconclusive.

\section{Results}

The test substance is considered to be mutagenic when the number of counted colonies exceeds the number of colonies in the negative controls by at least double and a relationship between dose and response can be observed ${ }^{6}$.

The results showed the numbers of revertant colonies which were treated with the test substance were less than twice of negative control with and 
without S9 Mix at all concentrations presented both in tables and figures. No dose-response relationship was obtained and no statistical procedure was used. The results in the Salmonella tester strains are presented in Table 1 and 2 and shown in Figure 2 and 3.

\section{Discussion}

In order to protect the human health, every single materials that need to be exposed to the human for example dental materials need to be confirmed safely. Based on International Standard, [2], all medical device shall be tested for genotoxicity except those made only from materials known to show no genotoxicity effect. There was approximately eight different in vitro assays are listed down for researchers to choose. However, the best two tests would be Organization for Economic Cooperation and Development (OECD) tests which were Salmonella typhimurium reverse mutation assay (471) and mammalian cytogenetic test $(473)^{8,9}$. In this study, the assay used was Salmonella typhimurium reverse mutation alone and it showed very significant findings.

Generally, a negative result can be defined using 4-5 strains. However, a single strain is sufficient to demonstrate a mutagenic response. Strains TA98, TA100, TA97 and TA1537 are always considered necessary. Besides, other strains such as TA102, TA104, TA1535 and TA1538 are also widely used.

Although all known assays can yield false-positive and false-negative results, experience shows that the combination of two different test methods is a reliable parameter for determining carcinogens which are a risk to human health ${ }^{5}$.

Concentrations of silver amalgam with a range from $313 \mathrm{mg}$ to $5000 \mathrm{mg}$ were chosen as standard values given by Mortelmans and Zeiger ${ }^{6}$, because this material has been previously approved to be out of cytotoxic effect and yet the $5000 \mathrm{mg}$ was the highest indicator value to respond to any genotoxic activity.

To ensure the comparability of results, the extraction temperature should preferably be $37+$ $0.5^{\circ} \mathrm{C}$ that mimicked the human body temperature and the time must be at least 24 hours ${ }^{10}$. Silver amalgam was dissolved into distilled water and not DMSO simply because DMSO solution is known to be highly cytotoxic.

This study involves the mixture of $\mathrm{S} 9$ as metabolic activation system because Salmonella typhymurium is only a prokaryote (basic cell living structure) and cannot represent the human being as a perfect model; therefore the addition of the exogenous liver enzyme could enhance the quality of the result.

The top agar is the most critical medium components in Ames test because it contains the trace amount of histidine $(0.05 \mathrm{mM})$ for limited growth of Salmonella strains. It also contains biotin at a concentration of $0.05 \mathrm{mM}$ which is in excess of what is needed for the growth of Salmonella strains. Too much histidine will cause heavy growth that may obsecure the revertant colonies ${ }^{6}$.

After 48 hours incubated, the revertants colonies of Salmonella typhymurium were counted by using Computerized Colony Analyzer (ProtoCol.). The specificity and sensitivity was fixed to $96 \%$ with $8 \mathrm{~cm}$ diameter of countered area. This measurement was done homogenously to all plates.

In the future, in vitro genotoxicity test which will preferably use more strains and mammalian cells as target should be carried out. In vivo genotoxicity test can be undertaken if scientifically indicated or in vitro test results indicate potential genotoxicity. A nanotechnology measurement technique hopefully can be applied to cut the tester material into more identical size according to reference doses.

\section{Conclusion}

In conclusion, these data were demonstrating as negative result and showing the test materials did not exhibit mutagenic activity and no dose-related increase in the number of revertant colonies under the present test conditions. Thus, the material was considered to have no genotoxic effect.

\section{Acknowledgment}

The authors are grateful to all technologists from Craniofacial Biology Laboratory, School of Dental Sciences, Universiti Sains Malaysia for their 
assistance, Dr. T.P. Kannan for his contribution of ideas and Dr. S. Radhakrishnan for his product contribution in this project.

\section{References}

1. Dunia Perwira Manufacturing Sdn. Bhd., "Silverfil Argentum $^{\circledR}$," 2006. Internet Available: http:// www.silverfil.com.

2. ISO (International Organization for Standardization) 10993-3, "Test for Genotoxicity, Carcinogenecity and Reproductive Toxicity," Biological Evaluation of Medical Devices - Part 3, 1992, pp 1-7.

3. Chauvel-Lebret DJ, Auroy P, Tricot-Doleux P, and Bonnaure-Mallet M. 2001. Evaluation of the Capacity of the SCGE Assay to Assess the Genotoxicity of Biomaterials. Biomaterials 22: 1795-1801.

4. Kirkpatrick CJ, Wagner $M$, Kohler $\mathrm{H}$, Bittinger $\mathrm{F}$, Otto $M$ and Klein CL. 1997. The Cell and Molecular Biological Approach to Biomaterial Research: A Perspective. J Mater. Sci., Mater. Medicine 8: 131-141.
5. Katzer H, Marquardt J, Westendorf J, Wening JV, and von Foerster G. 2002. PolyetheretherketoneCytotoxicity and Mutagenicity In Vitro. Biomaterials 23: 1749-1759.

6. Mortelmans K and Zeiger E. 2000. The Ames Salmonella/Microsome Mutagenicity Assay. Mutation Res 455: 29-60.

7. Chang HS. 2002. Cytotoxicity and Mutagenicity of Hydroxyappatite: An In Vitro Evaluation. Biomaterials : 13-17.

8. OECD (Organization for Economic Cooperation and Development) Guidelines. 1986. Introduction to the OECD Guidelines on Genetic Toxicology Testing and Guidance on the Selection and Application Assay," Guidelines on Genetic Toxicology: 1-17.

9. OECD (Organization for Economic Cooperation and Development) TG 471. 1997. Mutagenicity: Reverse Mutation Test Using Bacteria. Bacterial Reverse Mutation Test: 1-11.

10. Wening JV, Marquardt $\mathrm{H}$, Katzer A, Jungbluth JH, and Marquardt H. 1995. Cytotoxicity and Mutagenicity of Kevlar: An In Vitro Evaluation. Biomaterials 16: 337-340. 